\title{
Análisis crítico de un artículo: La rosiglitazona aumenta los eventos cardiovasculares a largo plazo
}

\author{
Ricardo Cruz U 1a, Luz María Letelier $\mathbf{S}^{1,2}$. \\ Critically appraised article: \\ Long-term risk of cardiovascular \\ events with rosiglitazone: \\ A meta-analysis \\ JAM A 2007; 298: 1189-95 \\ Sonal Singh, Yoon K. Loke, \\ Curt D. Furberg
}

Contex t: Recent reports of serious adverse events with rosiglitazone use have raised questions about whether the evidence of harm justifies its use for treatment of type 2 diabetes. Objective: To systematically review the long-term cardiovascular risks of rosiglitazone, including myocardial infarction, heart failure, and cardiovascular mortality. Data sources: We searched MEDLNE, the GlaxoSmithKline clinical trials register, the US Food and Drug Administration Web site, and product information sheets for randomized controlled trials, systematic reviews, and meta-analyses published in English through May 2007. Study selection: Studies were selected for inclusion if they were randomized controlled trials of rosiglitazone for prevention or treatment of type 2 diabetes, had at least 12 months of follow-up, and monitored cardiovascular adverse events and provided numerical data on all adverse events. Four studies were included after detailed screening of 140 trials for cardiovascular events. Data ex traction: Relative risks (RRs) of myocardial infarction, heart failure, and cardiovascular mortality were estimated using a fixed-effects meta-analysis of 4 randomized controlled trials ( $\mathrm{n}=14.291$, including 6.421 receiving rosiglitazone and 7.870 receiving control therapy, with a duration of follow-up of 1-4 years). Results: Rosiglitazone significantly increased the risk of myocardial infarction ( $\mathrm{n}=94 / 6421$ vs $83 / 7870 ; \mathrm{RR}, 1.42 ; 95 \%$ confidence interval [CI] 1.06-1.91; $\mathrm{P}=.02$ ) and heart failure ( $\mathrm{n}=102 / 6.421$ vs 62/7.870; $\mathrm{RR}, 2.09 ; 95 \% \mathrm{CI}, 1.52-2.88 ; \mathrm{P}$ $<001$ ) without a significant increase in risk of cardiovascular mortality ( $=59 / 6.421$ vs $72 / 7.870$; $R$, $0.90 ; 95 \% \mathrm{CI}, 0.63-1.26 ; \mathrm{P}=.53$ ). There was no evidence of substantial heterogeneity among the trials for these end points (I(2) $=0 \%$ for myocardial infarction, 18\% for heart failure, and $0 \%$ for cardiovascular mortality). Conclusion: Among patients with impaired glucose tolerance or type 2 diabetes, rosiglitazone use for at least 12 months is associated with a significantly increased risk of myocardial infarction and heart failure, without a significantly increased risk of cardiovascular mortality.

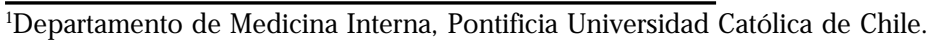

${ }^{2}$ Unidad de Medicina Basada en Evidencia

${ }^{\text {aResidente }}$
}

Correspondencia a: Dra. Luz M. Letelier. E mail: Imletel@med.puc.cl 


\section{CONCLUSIÓN DE LOS REVISORES:}

El uso de rosiglitazona por más de 12 meses, aumenta el riesgo de IAM e insuficiencia cardiaca, sin aumentar la mortalidad cardiovascular de pacientes con intolerancia a la glucosa o diabetes mellitus tipo 2 .

\section{PREGUNTA CĹNICA:}

¿El uso de rosiglitazona en pacientes que requieren hipoglicemiantes orales aumenta el riesgo de eventos cardiovasculares?

\section{ConTeXto:}

El uso de la rosiglitazona (una tiazolidinediona) fue aprobado por la FDA el año 1999 para el tratamiento de la diabetes mellitus tipo 2, debido a sus efectos benéficos en la reducción de la glicemia y en el control de otros factores de riesgo cardiovascular como la resistencia a la insulina y la presión arterial ${ }^{1}$. Posteriormente se comenzaron a reportar efectos adversos cardiovasculares serios con su uso, particularmente insuficiencia cardiaca ${ }^{2,3}$ e infarto al miocardio (IAM) ${ }^{4}$, lo que ha llevado a cuestionarse la seguridad del uso de este medicamento. El objetivo de la revisión sistemática analizada fue evaluar los efectos adversos cardiovasculares producidos por el uso a largo plazo de rosiglitazona.

\section{CARACTERÍSTICAS DEL ESTUDio:}

Diseño de estudio: Revisión sistemática de estudios clínicos randomizados (ECR) que evaluaron el uso de rosiglitazona para prevención o tratamiento de diabetes mellitus tipo 2 con al menos 12 meses de seguimiento y que reportaron eventos adversos cardiovasculares. Se realizó la búsqueda en MEDLNE, página web de la FDA y en reportes publicados y no publicados (fase 3 y 4) del registro de GlaxoSmithKline, sólo en inglés. Pacientes: Se incluyó 4 ECR con un total de 14.291 pacientes adultos con intolerancia a la glucosa o diabetes mellitus tipo 2. Intervención: Rosiglitazona $(\mathrm{n}=6.421)$. Comparación: Placebo u otro hipoglicemiante oral no tiazolidinediona ( $\mathrm{n}=7.870$ ). Outcome primario: Eventos cardiovasculares: IAM, insuficiencia cardiaca o mortalidad cardiovascular con seguimiento entre 1 y 4 años.

VALIDEZ:

Pregunta específica y focalizada

Búsqueda amplia y completa

Criterios de inclusión y exclusión claros y pertinentes a la pregunta

Evaluación de validez de estudios incluidos

Dos revisores independientes

Evaluación de heterogeneidad

Sí
$\mathrm{No}^{*}$
$\mathrm{Si}^{*}{ }^{*}$
Parcial
Sí
Sí

* La búsqueda no fue completa ya que usaron pocas bases de datos, sin embargo usaron algunas poco frecuentes que en este caso eran muy importantes.

** Sólo los de inclusión

***Sólo describen si eran ciegos o no 


\section{RESULTADOS:}

Se incluyeron 4 ECR, publicados hasta mayo de 2007, pero la calidad de los ECRs no fue bien evaluada. No se encontró heterogeneidad entre los estudios en ninguno de los 3 outcomes reportados.

\begin{tabular}{|lcccc|}
\hline O utcome & TEC (n) & TEE (n) & RR (IC 95\%) & N N H \\
\hline IAM & $1,05(83)$ & $1,46(94)$ & $1,42(1,06-1,91)$ & 244 \\
Insuficiencia cardiaca & $0,78(62)$ & $1,6(102)$ & $2,09(1,52-2,88)$ & 122 \\
Mortalidad cardiovascular & $(72)$ & $(59)$ & $0,90(0,63-1,26)$ & \\
\hline
\end{tabular}

TEC: tasa eventos grupo control (\%). TEE: tasa de eventos grupo experimental (\%). RR: riesgo relativo. NNH: número necesario para hacer daño. IC: intervalo de confianza.

\section{COMENTARIOS Y APLICACIÓN PRÁCTICA}

- Esta revisión sistemática cumple con la mayoría de los criterios de validez, pero no reporta adecuadamente la validez de los estudios incluidos, lo que habría sido importante, dado el pequeño número de estudios incluidos. Tampoco se reportaron claramente los criterios de exclusión y además la búsqueda fue incompleta. Los criterios de inclusión de los estudios fueron claros y consistentes con la pregunta planteada por los autores, el proceso de selección se hizo por 2 revisores y no se evidenció heterogeneidad entre los estudios para los outcomes evaluados.

- Este estudio demuestra que el uso de rosiglitazona aumenta al doble el riesgo de insuficiencia cardiaca y en $42 \%$ el riesgo de IAM, sin demostrar diferencias en la mortalidad cardiovascular. Estos resultados son concordantes con publicaciones previas $^{4,5}$, con la ventaja de usar estudios con mayor número de pacientes, sin heterogeneidad entre ellos y con recolección sistemática de los eventos cardiovasculares. Sin embargo, no se especifica el uso de medicamentos "cardioprotectores" (por ej: aspirina) que podrían reducir el riesgo basal.

- Si bien el riesgo absoluto de presentar eventos en cada grupo fue bajo, la diferencia entre los grupos es estadísticamente significativa para insuficiencia cardiaca y para IAM. A pesar de los riesgos absolutos de poca magnitud, estos resultados tienen gran relevancia si consideramos que la población susceptible de recibir rosiglitazona es enorme y similar a la población en nuestro medio.

- Así, según distintos riesgos basales los NNH para IAM pueden variar entre 74 y 822 pacientes:

$\begin{array}{ll}\text { Riesgo basal de IAM } & \text { NNH (95\% IC) } \\ 0,29 & 822(379-5.748) \\ 1,08 & 221(102-1.544) \\ 3,22 & 74(35-518)\end{array}$

En suma, dada la evidencia de que la rosiglitazona no sería superior a otros hipoglicemiantes orales en cuanto a control glicémico y mortalidad 6 , pero sí podría aumentar eventos cardiovasculares como IAM e insuficiencia cardiaca, parece razonable preferir otros hipoglicemiantes orales. 


\section{REFERENCIAS}

1. Qayyum $R$, Adomattyte J. A meta-analysis of the effect of thiazolidinediones on blood pressure. J Clin Hypertens (Greenwich) 2006; 8: 19-28.

2. SingH S, LOKe YK, FurBerg CD. Thiazolidinediones and heart failure: a teleo-analysis. Diabetes Care 2007; 30: 2148-53.

3. Gerstein HC, Yusuf S, Bosch J; Diabetes Reduction Assessment With Ramipril and Rosiglitazone Medication Trial Investigators. Effect of rosiglitazone on the frequency of diabetes in patients with impaired glucose tolerance or impaired fasting glucose: a randomised controlled trial. Lancet 2006; 368: 1096-105.

4. NISSEN SE, WolsKi K. Effect of rosiglitazone on the risk of myocardial infarction and death from cardiovascular causes. N Engl J Med 2007; 356: 2457- 71.

5. Giaxo smithKune. Glaxo SmithKline clinical trial register: study No. ZM2005/00181/01, Avandia Cardiovascular Event Modeling Project, and study No. HM2006/00497/00/WEUSRTP866, Coronary Heart Disease Outcomes in Patients Receiving Antidiabetic Agents. http://ctr.gsk.co.uk/Summary/ Rosiglitazone/III_CVmodeling.pdf. Accessed August 1, 2007.

6. Bolen S. Systematic Review: Comparative Effectiveness and Safety of Oral Medications for Type 2 Diabetes Mellitus. Ann Intern Med 2007; 147: 386-99. 